\title{
Primary Anorectal Malignant Melanoma Treated With Endoscopic Mucosal Resection
}

\author{
Jong Hoon Park ${ }^{1}$, Jeong Rok Lee ${ }^{1}$, Hyung Seok Yoon ${ }^{1}$, Tae Young Jung ${ }^{1}$, Eun Joo Lee ${ }^{1}$, Jong Gu Lim ${ }^{1}$ \\ Soon Young Ko ${ }^{1}$, Joon Ho Wang ${ }^{1}$, Jae Dong Lee ${ }^{1}$, Hye Young Kim ${ }^{2}$ \\ Departments of Internal Medicine, Anesthesiology and Pain Medicine', Konkuk University Chungju Hospital, Konkuk University School of \\ Medicine, Chungju, Korea
}

Anorectal melanoma is a rare neoplasm that accounts for less than $1-4 \%$ of anorectal malignant tumors. The main therapeutic modality for anorectal melanoma is surgical treatment, with abdominoperineal resection or wide local excision being the most common approaches. A 77-year-old male with a history of cerebral infarction and hypertension presented with anal bleeding. Here, we report a case of anorectal melanoma treated by endoscopic mucosal resection with adjuvant interferon therapy rather than surgical resection. The patient has been disease-free for 5 years after endoscopic treatment. (Intest Res 2015;13:170-174)

Key Words: Anorectal melanoma; Endoscopic mucosal resection; Interferon-alpha; Abdominoperineal resection; Wide local excision

\section{INTRODUCTION}

Anorectal melanoma (ARM) is a rare neoplasm with an extremely poor prognosis. It represents less than $1 \%$ of all melanomas and accounts for less than $4 \%$ of anal malignancies. ${ }^{1}$ ARM is documented as the third most common location of melanoma, after cutaneous and ocular. Patients with ARM often present with local symptoms such as rectal bleeding (55\%), rectal masses (34\%), and anal pain (13\%). ${ }^{2}$ ARM has been shown to result in worse prognoses than cutaneous melanoma, with a median survival of 24 months and a 5 -year survival rate of $10 \% .^{3}$ In the rectum, melanocytes are located at the anal transitional and squamous zones. Most ARMs arise from the dentate line, and $65 \%$ are located within the anal canal or at the anal verge. ${ }^{4}$ ARM

Received May 15, 2014. Revised July 5, 2014

Accepted August 11, 2014

Correspondence to Jeong Rok Lee, Department of Internal Medicine, Chungju Hospital, Konkuk University School of Medicine, 82 Gugwon-daero Chungju 380-704, Korea. Tel: +82-43-840-8631, Fax: +82-43-840-8973,

E-mail: flyingbass93@gmail.com

Financial support: None. Conflict of interest: None. spreads along submucosal planes, and is often too widespread for complete resection at the time of diagnosis and almost all patients die because of metastases. ${ }^{5}$ Although there are various treatment strategies for ARM, surgical treatment remains the main therapeutic modality due to its relative rarity, with abdominoperineal resection (APR) and wide local excision (WLE) being performed in most cases. The role of adjuvant chemotherapy and immunotherapy in the treatment of ARM is yet to be established. However, there is no consensus on which surgical approach is favorable. Several studies have reported cases of long-term survival with treatment consisting of only local surgical excision, ${ }^{6,7}$ and there have been other cases removed by endoscopic mucosal resection (EMR) alone in Japan. ${ }^{8}$ Here, we present a case of ARM treated with EMR with adjuvant interferon therapy. The patient has survived for 5 years without distant metastases.

\section{CASE REPORT}

A 77-year-old man was admitted to our emergency department with anal bleeding. He had a 4-month history of

\footnotetext{
(c) Copyright 2015. Korean Association for the Study of Intestinal Diseases. All rights reserved.

This is an Open Access article distributed under the terms of the Creative Commons Attribution Non-Commercial License (http://creativecommons.org/licenses/by-nc/3.0)

which permits unrestricted non-commercial use, distribution, and reproduction in any medium, provided the original work is properly cited.
} 
anal bleeding and recent aggravation. His medical history included cerebral infarction with right sided weakness and hypertension. On admission, he was hemodynamically stable and his hemoglobin level was $11.4 \mathrm{~g} / \mathrm{dL}$. His abdomen was soft and non-tender. Digital rectal examination revealed a palpable mass adjacent to the anal verge. Urgent colonoscopy demonstrated a dark-colored, polypoid mass with a diameter of $1.5 \mathrm{~cm}$ and oozing hemorrhage adjacent to the anal verge. Because of continuous bleeding, polypectomy was performed with a snare (Fig. 1). Analysis of the resected specimen, measuring $1.5 \times 1.2 \mathrm{~cm}$ in size, revealed a black-pigmented solid tumor with a short stalk (Fig. 2A). Microscopic findings revealed diffuse infiltration of round or spindle-shaped tumor cells, and the contained melanin and tumor cells were immunohistochemically positive for Human Melanin Black-45 (HMB-45) (Fig. 2B and C). After polypectomy, the resection margin contained positive tumor cells and it was difficult to confirm the depth of tumor invasion because of the presence of a short stalk. Neither lymphatic nor vascular invasion were noted. To evaluate systemic metastases, a fluorine-18-fluorodeoxyglucose (FDG) PET/ CT scan was performed after initial polypectomy. The PET/ CT scan demonstrated increased FDG uptake in the distal rectum (Fig. 3A). Though this finding might have indicated a remnant lesion of ARM, there was also the possibility of false positivity associated with initial polypectomy. However, the PET/CT scan showed no positive regional lymph nodes or systemic metastases (Fig. 3B).

We recommended surgical treatment, such as APR or WLE, to the patient and his family because of the poor prognosis of malignant melanoma and the positive resection margin. However, they refused surgery due to the patient's
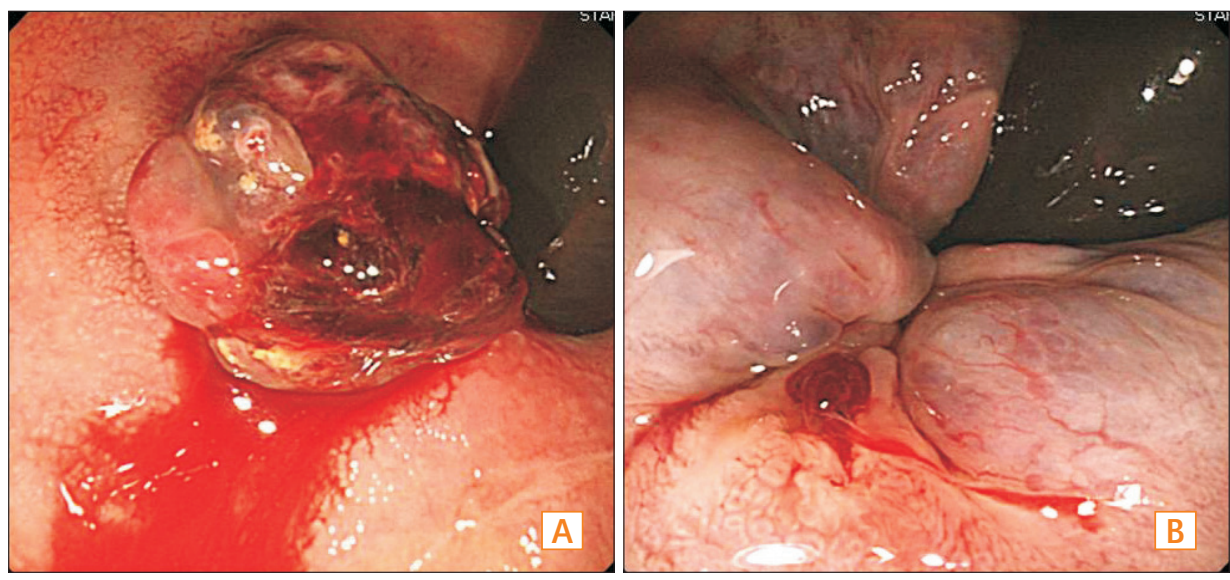

Fig. 1. Serial colonoscopic findings. (A) Colonoscopic view demonstrates a dark polypoid lesion with oozing hemorrhage about $1.5 \mathrm{~cm}$ in size adjacent to the anal verge. (B) After polypectomy, oozing hemorrhage is stopped.
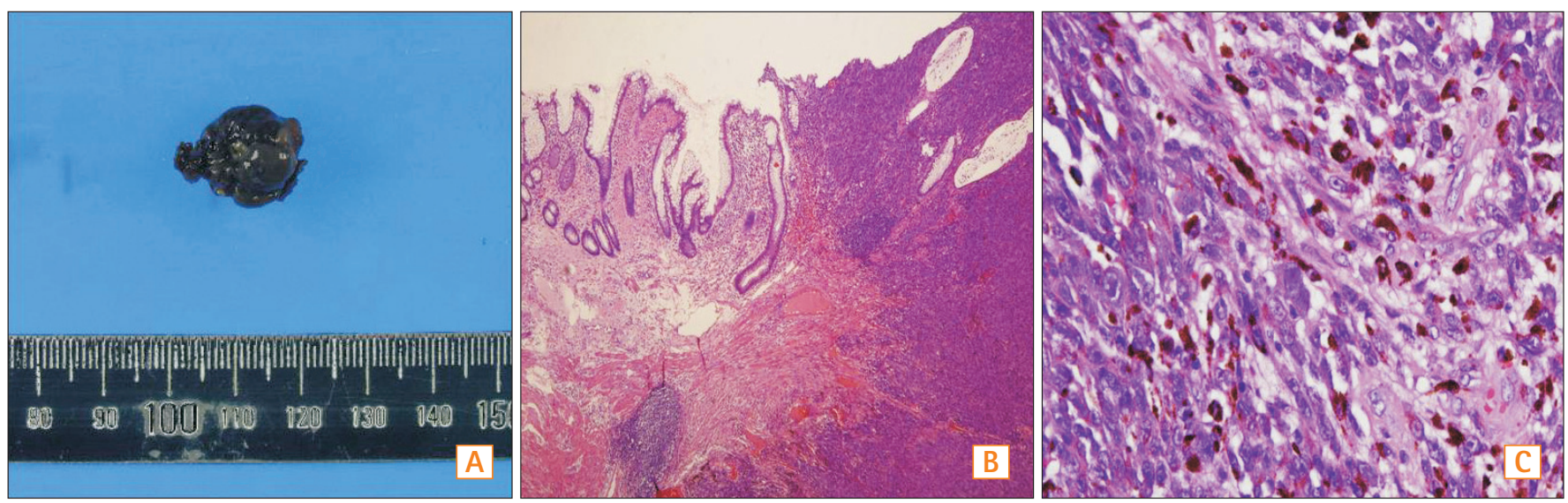

Fig. 2. Pathologic findings. (A) A gross view of the resected specimen, measuring $15 \times 12 \mathrm{~mm}$, shows a dark, black-pigmented solid tumor with a short stalk. (B) Microscopic findings show diffuse infiltration of round or spindle-shaped tumor cells with lymphocytes (HEEE, x40). (C) Immunohistochemically, tumor cells are positive for Human Melanin Black-45 (HMB-45 staining, x400). 

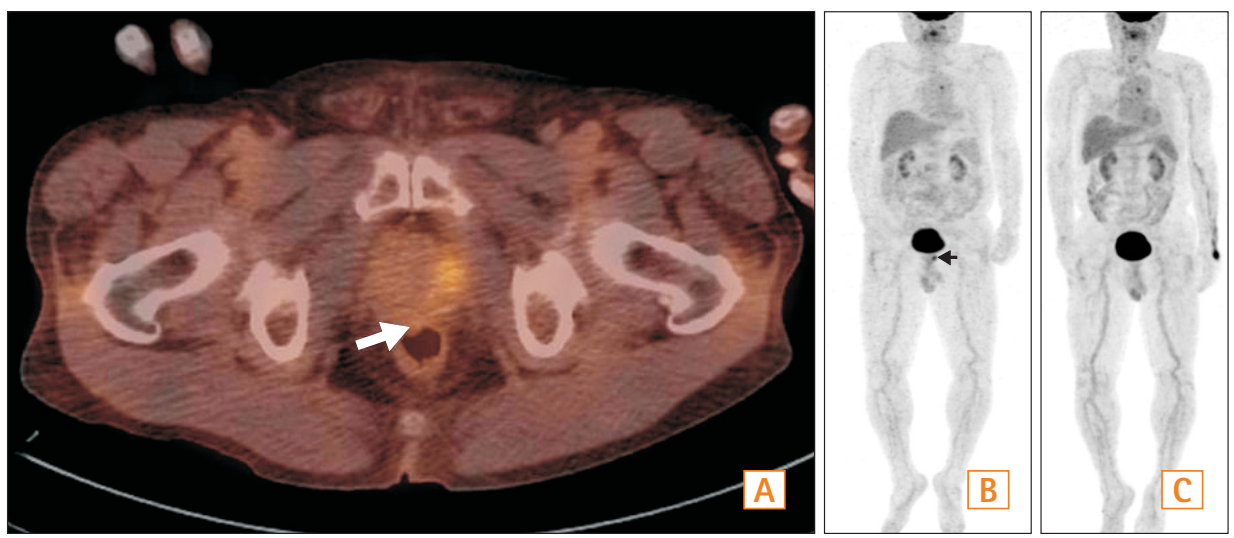

Fig. 3. Fluorine-18-fluorodeoxyglucose PET/CT findings. (A) After polypectomy, a PET/CT scan of abdomen reveals a mild hypermetabolic lesion at the anorectal junction (Maximum standardized uptake values, 2.3; white arrow). (B) There is neither lymph node nor systemic metastases. Hypermetabolic lesion at the anorectal junction is observed (arrow head). (C) Five years after initial therapy, PET/CT scan does not demonstrate the hypermetabolic lesion.
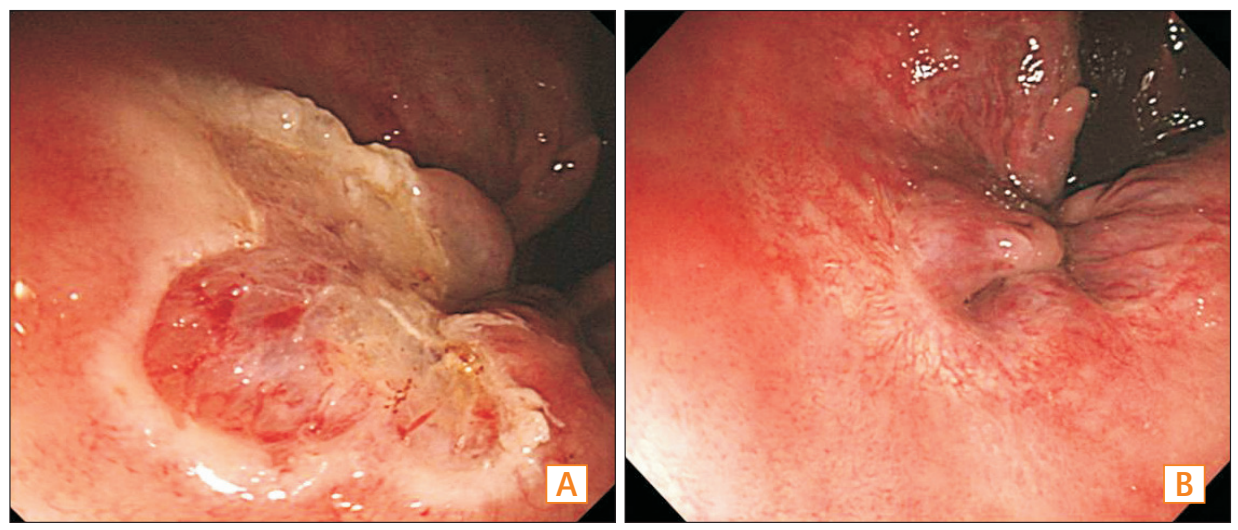

Fig. 4. Sigmoidoscopic findings. (A) Additional endoscopic mucosal resection (EMR) is performed with three pieces at the site of previous polypectomy. (B) Sigmoidoscopic view reveals scar change at previous EMR site 5 years after initial therapy.

old age and general weakness secondary to cerebral infarction. Therefore, we designed and performed additional EMR for the positive resection margin (Fig. 4A). Tumor cells were not observed in the resected specimen. Since there was a positive resection margin after the first polypectomy, and difficulty in evaluating the depth of tumor invasion, we decided on and recommended adjuvant therapy. The patient was treated with interferon $\alpha 2 \mathrm{~b}$ injection, $20 \times 10^{6} \mathrm{IU} /$ $\mathrm{m}^{2}$, five times per week for four weeks, then $10 \times 10^{6} \mathrm{IU} / \mathrm{m}^{2}$, three times per week for 24 weeks subcutaneously. During the 5-year follow-up period, the patient was asymptomatic and abdominal CT scans and sigmoidoscopic examinations at 3-6-month intervals revealed no evidence of recurrence (Fig. 3C and 4B).

\section{DISCUSSION}

Melanoma of the anorectal region is a relatively rare neoplasm with a poor prognosis. The overall 5 -year survival rates in patients with ARM range from $4-31 \%$ even if radical surgery and chemotherapy are performed, while median survival varies from $16-28$ months. ${ }^{3,9,10}$ Treatment strate- gies have varied, from the radical APR to the conservative WLE, but the main controversy has been whether APR is needed or WLE is adequate for complete treatment. APR, although associated with a high rate of morbidity, has long been thought to be the best means of management for ARM. In 1997, however, there was a paradigm shift after two major studies found minimal improvements in survival. Retrospective studies were performed, which looked at 135 patients with anal melanoma. ${ }^{2,11}$ Patients in both studies had uniformly poor survival rates and the APR group had a longer survival rate, but this was not statistically significant. ${ }^{11}$ In a study from Thibault et al., ${ }^{2} 37$ patients had curative resection but no significant survival difference was found between WLE and APR when comparing disease stage and 5-year survival. ${ }^{12}$ Before 1997, 70\% of all patients underwent APR, and after 1997, 80\% would undergo WLE. ${ }^{13}$ Furthermore, because the completion of lymphadenectomy in APR may not affect survival, ${ }^{13}$ WLE with or without adjuvant therapy seems to offer good locoregional control without reducing the survival rate, and may be a therapeutic modality for patients with small, superficial ARMs. ${ }^{14}$ APR should be offered for patients with locally advanced disease or as a salvage 
therapy following recurrence. ${ }^{14,15}$

There is no current adjuvant therapy dictated as the standard of care in ARM, particularly in cases involving metastatic disease. There have not been any randomized controlled trials to study this specifically. However, it is clear that chemotherapy alone without surgery provides no benefit. ${ }^{12}$ Radiation therapy has not been shown to provide any benefit, except occasionally for palliative care in cases of unresectable tumors. ${ }^{16}$ Some patients with metastatic melanomas have been shown to respond to therapy with interferon $\alpha$ and interleukin-2, suggesting that metastatic melanoma is susceptible to immune assault. ${ }^{16}$

EMR is not a "wide" but a "local excision" method. In Japan, EMR for malignant melanoma in the gastrointestinal tract has been performed in only five cases, and there are no reported cases from other countries. ${ }^{8}$ Relatively long-term survival was achieved in all cases ( $>6$ years). Among these cases, however, two were melanomas in the esophagus, another two required additional surgical treatment and chemotherapy, and the other involving a 85-year-old male with a 22x18 mm-sized tumor and submucosal invasion, needed repeated EMRs because local recurrence had occurred four times previously. ${ }^{8}$ In cases of ARM in which a shallow depth of the invasion and a small tumor size, long-term survival is expected even if less invasive surgery such as EMR is performed. ${ }^{8}$ For the assessment of invasion depth in ARM, the preferred modality is MRI. Anatomical and functional diagnostic imaging, such as CT, MRI, and PET/CT scans are available to assess lymph node involvement and to exclude or confirm the presence of distant metastases, as well as loco-regional recurrences. ${ }^{17}$ Until now, however, PET/CT scans have been reported rarely in cases of ARM for the detection of distant metastasis or recurrent disease. ${ }^{17}$

In the present case, EMR with adjuvant interferon therapy lead to 5-year disease free survival. Even though EMR in this case was not the ideal treatment modality and it the depth of tumor invasion was unclear, there was no local recurrence, distant metastases, or additional intervention needed after the first treatment for 5 years. Worse histological prognoses have been found to be associated with tumor thickness, tumor necrosis, and perineural invasion. ${ }^{2,11}$ In our case, there was no necrosis and perineural invasion, and though it was difficult to assess the depth of invasion exactly, we suspected superficial invasion confined to the submucosal layer because EMR removes only mucosa and superficial portions of the submucosa. The resection margin of the tumor in the first polypectomy was positive, but negative in the second EMR. However, because the resection margin was positive and the depth of tumor invasion was not clear in the initial polypectomy, we needed adjuvant interferon therapy after polypectomy and EMR.

In conclusion, ARM has a poor prognosis and needs surgical treatment. Nevertheless, if there is a shallow depth of invasion and the general condition of the patient is not good enough to justify surgery, less invasive surgical methods such as EMR with or without adjuvant therapy may be performed.

\section{REFERENCES}

1. Klas JV, Rothenberger DA, Wong WD, Madoff RD. Malignant tumors of the anal canal: the spectrum of disease, treatment, and outcomes. Cancer 1999;85:1686-1693.

2. Thibault C, Sagar P, Nivatvongs S, Ilstrup DM, Wolff BG. Anorectal melanoma-an incurable disease? Dis Colon Rectum 1997;40:661-668.

3. Solaz Moreno E, Vallalta Morales M, Silla Búrdalo G, Cervera Miguel JI, Díaz Beveridge R, Rayón Martín JM. Primary melanoma of the rectum: an infrequent neoplasia with an atypical presentation. Clin Transl Oncol 2005;7:171-173.

4. Row D, Weiser MR. Anorectal melanoma. Clin Colon Rectal Surg 2009;22:120-126.

5. Kohli S, Narang S, Singhal A, Kumar V, Kaur O, Chandoke R. Malignant melanoma of the rectum. J Clin Imaging Sci 2014;4:4.

6. Malik A, Hull TL, Milsom J. Long-term survivor of anorectal melanoma: report of a case. Dis Colon Rectum 2002;45:14121415; discussion 1415-1417.

7. Bullard KM, Tuttle TM, Rothenberger DA, et al. Surgical therapy for anorectal melanoma. J Am Coll Surg 2003;196:206-211.

8. Tanaka S, Ohta T, Fujimoto T, Makino Y, Murakami I. Endoscopic mucosal resection of primary anorectal malignant melanoma: a case report. Acta Med Okayama 2008;62:421-424.

9. Pessaux P, Pocard M, Elias D, et al. Surgical management of primary anorectal melanoma. Br J Surg 2004;91:1183-1187.

10. Weinstock MA. Epidemiology and prognosis of anorectal melanoma. Gastroenterology 1993;104:174-178.

11. Brady MS, Kavolius JP, Quan SH. Anorectal melanoma. A 64year experience at Memorial Sloan-Kettering Cancer Center. Dis Colon Rectum 1995;38:146-151.

12. Yap LB, Neary P. A comparison of wide local excision with abdominoperineal resection in anorectal melanoma. Melanoma Res 2004;14:147-150.

13. Yeh JJ, Shia J, Hwu WJ, et al. The role of abdominoperineal resection as surgical therapy for anorectal melanoma. Ann Surg 2006;244:1012-1017. 
14. Belli F, Gallino GF, Lo Vullo S, Mariani L, Poiasina E, Leo E. Melanoma of the anorectal region: the experience of the National Cancer Institute of Milano. Eur J Surg Oncol 2009;35:757-762.

15. Ramakrishnan AS, Mahajan V, Kannan R. Optimizing local control in anorectal melanoma. Indian J Cancer 2008;45:13-19.
16. Stefanou A, Nalamati SP. Anorectal melanoma. Clin Colon Rectal Surg 2011;24:171-176.

17. Falch C, Stojadinovic A, Hann-von-Weyhern C, et al. Anorectal malignant melanoma: extensive 45-year review and proposal for a novel staging classification. J Am Coll Surg 2013;217:324335. 